Wilfrid Laurier University

Scholars Commons @ Laurier

Physics and Computer Science Faculty

Publications

Physics and Computer Science

1993

\title{
Hyperradiance from Phase-Locked Soliton Oscillators
}

Niels Grønbech-Jensen

Los Alamos National Laboratory

James A. Blackburn

Wilfrid Laurier University, jabjabjab@cogeco.ca

Follow this and additional works at: https://scholars.wlu.ca/phys_faculty

\section{Recommended Citation}

Grønbech-Jensen, Niels and Blackburn, James A., "Hyperradiance from Phase-Locked Soliton Oscillators" (1993). Physics and Computer Science Faculty Publications. 48.

https://scholars.wlu.ca/phys_faculty/48

This Article is brought to you for free and open access by the Physics and Computer Science at Scholars Commons @ Laurier. It has been accepted for inclusion in Physics and Computer Science Faculty Publications by an authorized administrator of Scholars Commons @ Laurier. For more information, please contact scholarscommons@wlu.ca. 


\title{
Hyperradiance from Phase-Locked Soliton Oscillators
}

\author{
Niels Grønbech-Jensen \\ Theoretical Division, Los Alamos National Laboratory, Los Alamos, New Mexico 87545 \\ James A. Blackburn \\ Department of Physics and Computing, Wilfrid Laurier University, Waterloo, Ontario, Canada N2L $3 C 5$
}

(Received 11 November 1992)

\begin{abstract}
We show that the power emitted from phase-locked soliton oscillators can exceed the upper limit given by the theory for superradiance from phase-locked point oscillators. As an experimentally relevant example, we consider two magnetically coupled long Josephson junctions, operated in singlefluxon modes. Analytical results indicate that the coupling between the two oscillators enables the phase-locked modes to localize in space thereby creating the possibility of an enhancement of the power level beyond the point-oscillator limit. Good agreement is found between analytical results and numerical simulations, and with recently published experimental data.
\end{abstract}

PACS numbers: $42.50 . \mathrm{Fx}, 03.40 . \mathrm{Kf}, 42.60 . \mathrm{By}, 74.50 .+\mathrm{r}$

Josephson effect devices have presented intriguing possibilities as tunable oscillators since their discovery nearly thirty years ago. A significant practical difficulty has always been the relatively small amount of radiation which can be coupled out of such an oscillator. A potential escape from this dilemma appeared more than two decades ago, when it was realized [1] that coherent arrays of Josephson oscillators should, in principle, exhibit "superradiance" for which the emitted power would scale as the square of the number of sources in the array. The first observation of superradiant emission, for a pair of junctions, was reported by Finnegan and Wahlsten [2]. The expected coherence-induced narrowing of the linewidth was confirmed by Varmazis et al. [3], again for a system of two coupled microbridges. Larger arrays involving 10 junctions [4] and even 99 junctions [5] have been studied.

Long Josephson junctions sustain soliton oscillations, and have also been the focus of considerable attention because of their possible use as sources of radiation. Phase locking has been observed for coupled pairs of long junctions [6], as well as in larger networks of 7 elements [7] or even 100 elements of these devices [8]. It has been supposed, in analogy with the behavior of arrays of small junctions, that a coherent state of $N$ soliton oscillators would likewise lead to radiated power enhancement by the superradiant factor $N^{2}$. Two recent publications reported surprising contradictions to this idea. Holst et al. [9] experimentally measured the radiation from a coupled pair of long Josephson junctions, and observed an output power nearly 1.5 times the expected value. For two coherent fluxon oscillators, Cirillo et al. [10] also experimentally found "that there exists even more power coupled with respect to what is expected in terms of the superradiant model."

This excess superradiant (hyperradiant) power will be shown to have its origin in the additional spatial degree of freedom inherent in a soliton oscillator. The positional coordinate permits adjustments in the wave forms for each member of an array beyond the simple phase alignment associated with coherence. It will be demonstrated that the superradiant limit $N^{2}$ does not therefore apply to coherent soliton oscillators, and, more remarkably, that no theoretical upper limit exists for the power enhancement.

The equations of motion for the field variables in the two long Josephson junctions (LJJs) are [11-13]

$$
\phi_{i, x x}-\phi_{i, t t}-\sin \phi_{i}=\alpha \phi_{i, t}-\beta \phi_{i, x x t}-\eta_{i}+\Delta \phi_{j, x x},
$$

where $i=1,2$ and $i \neq j$. The phases, $\phi_{1}$ and $\phi_{2}$, represent the quantum mechanical phase difference over each of the junctions. The spatial dimension, $x$, is normalized to the characteristic Josephson length, $\lambda_{J}$, and the time dimension, $t$, is normalized to the inverse plasma frequency, $\omega_{p}^{-1}$, of the junctions. The terms proportional to $\alpha$ and $\beta$ represent dissipative effects in the system. The first arises from damping due to the tunneling of normal electrons, the voltage across junction $i$ being $\sim \phi_{i, t}$. The second includes two types of damping: one from the transport of quasiparticles in the surfaces of the superconductors [14] and the other from radiation to the surroundings by the magnetic field distribution in the junction [15]. Both of these terms are proportional to $\phi_{i, x x t}$. The normalized bias current density in junction $i$ is denoted by $\eta_{i}$. Finally, the magnetic coupling is given by the parameter $\Delta$. For simplicity, we have chosen to keep the characteristic space and time constants as well as the damping parameters identical for the two junctions. This choice does not affect the principles discussed in this paper, but it enables us to make some analytical considerations.

A typical mode relevant for LJJs is the kink soliton solution to the unperturbed sine-Gordon equation [lefthand side of Eq. (1)],

$$
\phi_{i}=4 \tan ^{-1} \exp \left[\gamma\left(u_{i}\right)\left(x-u_{i} t\right)\right]
$$


where $\gamma(u)=\left(1-u^{2}\right)^{-1 / 2}$ is the inverse Lorentz contraction of a kink with the velocity $u$. The above solution is only valid for an infinite system. In reality, systems are of finite length and are governed by suitable boundary conditions. The most common are the open ones, $\phi_{i, x}(0)=\phi_{i, x}(L)=0$, where $L$ is the length of the terminated system.

The emitted power from the system can be defined in different ways according to the detection system. We consider three different emitted powers, defined as follows:

$$
\begin{aligned}
& P_{a}=\delta_{a} \int\left(\sum_{i} \phi_{i, x t}\right)^{2} d x \\
& P_{b}=\delta_{b} \int\left(\sum_{i} \phi_{i, t}\right)^{2} d x \\
& P_{c}=\delta_{c}\left(\sum_{i} \phi_{i, t \mid x=0}\right)^{2} .
\end{aligned}
$$

The definition of $P_{a}$ reflects the power of the magnetic far field radiated from the system, since the emitted power in the far-field approximation is proportional to the square of the time derivative of the magnetic field, which is itself proportional to $\sum \phi_{i, x}$. As defined, $P_{b}$ measures the electric power, which is proportional to the square of the voltage. The third definition, $P_{c}$, describes the result of a measurement performed through a boundary of the system if the open boundary conditions are applied. The parameters $\delta_{j}$ denote the coupling to the surroundings.

As was shown in Refs. $[12,13]$ two kinks of different, but coupled, sine-Gordon systems may form a bound state in parts of the parameter space. As a result of the choice of equal characteristic parameters, we may for any $\left|\eta_{1}+\eta_{2}\right|<2$ define a range in $\eta_{1}-\eta_{2}$ for which the two kinks are locked in their motion [13]. The center of this locking range is at the bias point, $\eta_{1}=\eta_{2}$.

Let us now calculate the emitted power from the coupled system if the two individual systems are biased equally and both contain one kink soliton. In this case, the two fields may be described by a single phase, $\psi \equiv \phi_{i}$, obeying the equation

$$
(1-\Delta) \psi_{x x}-\psi_{t t}-\sin \psi=\alpha \psi_{t}-\beta \psi_{x x t}-\eta
$$

This equation has the kink solution

$$
\psi=4 \tan ^{-1} \exp \left[\gamma\left(\frac{u}{\sqrt{1-\Delta}}\right) \frac{x-u t}{\sqrt{1-\Delta}}\right] .
$$

It is important to note here that not only has the coupling between the two systems contracted the kinks by a factor, $(1-\Delta)^{-1 / 2}$, but it has also decreased the asymptotic velocity of the kinks by the same factor. The emitted power from an infinite system of two equally biased LJJs, each operated in the one-fluxon mode, is then given by

$$
\begin{aligned}
P_{a}^{(12)} & =4 \delta_{a} \int \psi_{x t}^{2} d x \\
& =4 \delta_{a} \frac{8 u^{2}}{3(1-\Delta)^{3 / 2}} \gamma^{3}\left(\frac{u}{\sqrt{1-\Delta}}\right), \\
P_{b}^{(12)} & =4 \delta_{b} \int \psi_{t}^{2} d x=4 \delta_{b} \frac{8 u^{2}}{\sqrt{1-\Delta}} \gamma\left(\frac{u}{\sqrt{1-\Delta}}\right) .
\end{aligned}
$$

For the same system, with only junction 1 containing a fluxon, we obtain the powers

$$
\begin{aligned}
P_{a}^{(1)} & \simeq \delta_{a} \int\left(\phi_{1, x t}^{2}+2 \phi_{1, x t} \phi_{2, x t}\right) d x \\
& =\delta_{a} \frac{8 u^{2}}{3} \gamma^{3}(u)\left[1+6 \Delta \gamma^{2}(u) I\right], \\
P_{b}^{(1)} & \simeq \delta_{b} \int\left(\phi_{1, t}^{2}+2 \phi_{1, t} \phi_{2, t}\right) d x \\
& =\delta_{b} 8 u^{2} \gamma(u)\left[1+2 \Delta \gamma^{2}(u) J\right]
\end{aligned}
$$

Here we have taken into account the wave profile for junction $2\left(\phi_{2}\right)$, resulting from the kink in junction $1\left(\phi_{1}\right)$. In the linear approximation (small $\Delta$ ) this is given by [12]

$$
\phi_{2}=2 \Delta \gamma^{2}(u)[Z \cosh Z-\sinh Z \ln (2 \cosh Z)],
$$

where $Z=\gamma(u)(x-u t)$. The numbers $I$ and $J$ are given by

$$
\begin{aligned}
I & =\int_{0}^{\infty}\left[\tanh ^{2} z \operatorname{sech}^{2} z+\tanh ^{2} z \ln (2 \cosh z)-z \tanh z\right] d z \\
& \approx 0.1558 \\
J & =\int_{0}^{\infty}\left[\operatorname{sech}^{2} z-\ln (2 \cosh z)-z \tanh z\right] d z \\
& \approx 0.1775 .
\end{aligned}
$$

Defining the ratio, $\kappa$, between the emitted power from the two oscillators operated synchronously and operated individually, we find the maximal power ratios, $\kappa_{j}^{*}$, from Eqs. (8) - (11) for $\eta_{1}=\eta_{2}$, to be

$$
\begin{aligned}
\kappa_{a}^{*} & =P_{a}^{(12)} / P_{a}^{(1)} \\
& \simeq 4\left(\frac{1-u^{2}}{1-u^{2}-\Delta}\right)^{3 / 2}\left[1+6 \Delta \gamma^{2}(u) I\right]^{-1}, \\
\kappa_{b}^{*} & =P_{b}^{(12)} / P_{b}^{(1)} \\
& \simeq 4 \sqrt{\frac{1-u^{2}}{1-u^{2}-\Delta}}\left[1+2 \Delta \gamma^{2}(u) J\right]^{-1} .
\end{aligned}
$$

From these expressions, valid at the center of the locking range of the infinite system, we find that the maximal ratio between the power in the phase-locked mode and the individual mode is always larger than 4. Furthermore, we note that as the soliton velocity (frequency) approaches the asymptote $u / \sqrt{1-\Delta}$, the ratios, $\kappa_{j}^{*}$, diverge for both definitions of the power. As a result of the limited power supply to the system, $\kappa_{j}$ cannot in practice increase without limit. Dynamically, this is ensured by the fact that the unperturbed wave profiles of the solitons do not follow the expressions Eqs. (2) and (7) when the system is strongly perturbed [13]. 
We have performed numerical experiments on the system defined by Eq. (1) for different boundary conditions and system parameters. As experimentally relevant system parameters we have chosen $L=5$ and $\alpha=0.05$ throughout the paper. The numerical procedure for determining the power ratios was the following. For a given set of system parameters and boundary conditions, we measured the powers as they are defined by Eqs. (3)(5) for the case of both systems $(i=1,2)$ containing a kink soliton as well as for the case where only one system $(i=1)$ contained a kink. Two different types of bias scans were performed. One was to vary $\eta_{1}+\eta_{2}$ for $\eta_{1}-\eta_{2}=0$, and the other was to vary $\eta_{1}-\eta_{2}$ for fixed values of $\eta_{1}+\eta_{2}$. In all cases we measured the normalized voltage of the individual junctions in order to make the power ratios, $\kappa_{j}$, for $P_{i}^{(12)}$ and $P_{i}^{(1)}$ measured at the same voltage (soliton speed). The numerical simulations were performed for both open and periodic boundary conditions and for different values of the damping parameter, $\beta$. However, in order to simplify the discussion, we have elected to present only the results for $\beta=10^{-3}$ and open boundary conditions. We note that all the results not shown in this paper exhibit the same characteristics as the results which are presented now.

In Fig. 1 we show the dynamically evaluated power ratios for the three different definitions of the power [i.e., Eqs. (3)-(5)], for different values of the coupling parameter, $\Delta$. The insets show the power ratio as $\eta_{1}-\eta_{2}$ is varied from the center $\left(\eta_{1}-\eta_{2}=0\right)$ of the locking range to the edge. These data are for the fixed value, $\eta_{1}+\eta_{2}=1.2$. As is obvious from the figures, the power ratios have their maxima in the center of the locking range and these maxima are all larger than 4 , which is the theoretical superradiant limit for a pair of phase-locked point oscillators. If we follow the power ratios, $\kappa_{j}^{*}$, in the center of the locking range $\left(\eta_{1}-\eta_{2}=0\right)$ as a function of the common bias, $\eta_{1}=\eta_{2}$, we find that the values are always larger than 4 and increase as the bound state of kinks approaches their rescaled asymptotic velocity, $\sqrt{1-\Delta} \approx \omega L / \pi$, where $\omega$ is the frequency of the kink motion in the terminated sine-Gordon systems. We have here chosen to show the power ratios as a function of the kink frequency, since this way of displaying the results also reveals the decreasing asymptotic frequency with increasing coupling

FIG. 1. The power ratios, $\kappa_{j}$, averaged over a complete number of periods of motion, for different parameter sets. The insets show the power ratios as the system is taken through the locking range for different values of the coupling parameter $\Delta$. The maximal value of the power ratios, $\kappa_{j}^{*}$, is shown as a function of the soliton frequency $\omega$. System parameters are $L=5, \alpha=0.05$, and $\beta=10^{-3}$. The open boundary conditions are used and all results shown are from numerical simulations on Eq. (1). (a) The power definition Eq. (3). (b) The power definition Eq. (4). (c) The power definition Eq. (5).
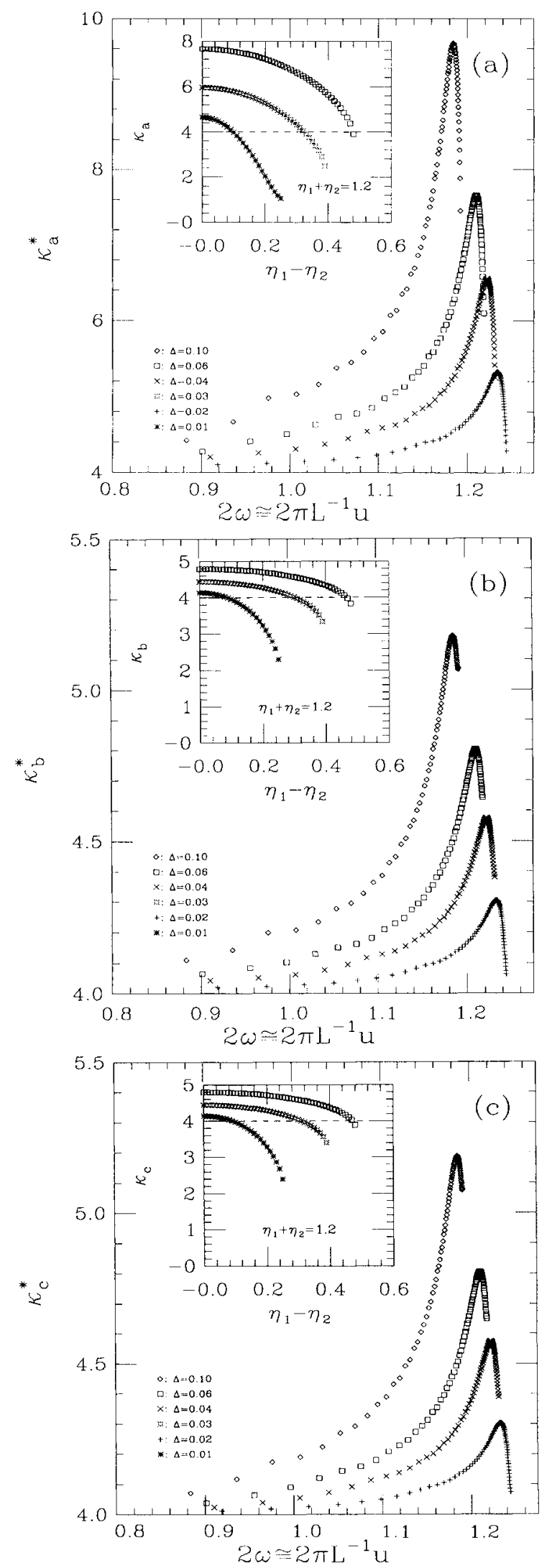
parameter. When the kink frequency gets very close to the asymptote, the power ratios tend to decrease rapidly. This is caused by the kink deformations in a strongly perturbed system, for which the adiabatic perturbation method used above is not applicable. However, we do find very close qualitative agreement between the results of the analytical treatment, Eqs. (13) and (14), and the numerical results, shown in Figs. 1(a) and 1(b). First of all, we see that the power ratios are all larger than 4 when the system is operated near the center of the locking range. Second, we find the singularitylike behavior as the kink frequency approaches its asymptote. Finally we find that the ratio, $\kappa_{a}$, between the magnetic power emissions is consistently larger than the ratio, $\kappa_{b}$, between the electric powers. Another trend, apparent in Fig. 1, is the decreasing power ratio (for all the power definitions) as the coupling parameter is decreased. From the figures we find that the maximal ratios, $\kappa_{j}^{*}$, approach the superradiant limit, $N^{2}$, for $\Delta \rightarrow 0$. This is also consistent with the analytic results Eqs. (13) and (14), which give $\kappa_{j}^{*} \rightarrow 4_{+}$for $\Delta \rightarrow 0$. This limit shows that the phase-locking under extremely weak coupling yields the superradiant power ratio. For stronger coupling, we not only get better phase-locking between the oscillators, but we also experience the internal redistribution of the individual modes, responsible for the hyperradiance.

As noted earlier, the phenomenon of excessive superradiance from soliton oscillators has been observed experimentally [9, 10]. In Ref. [9] the experimental system consisted of arrays of two inductively coupled LJJs-a configuration very similar to the model studied in this paper. The results in Ref. [9] showed emitted power levels nearly 1.5 times the value of the point-oscillator limit. We note that the experimental setup did not reveal exactly what type of power was measured. However, we have seen that all three definitions of the power measurement in this paper give rise to hyperradiance, and we may therefore predict that the emitted power level from an array of $N$ phase-locked LJJs, operated at a given frequency, can exceed $N^{2}$ times the power level from a single oscillator operated at the same frequency. Furthermore, the ratio between the power from the locked states, and from the individual oscillators should increase dramatically when the soliton speed is close to the asymptotic value.
In conclusion, we have shown that the superradiant limit of the emitted power from phase-locked point oscillators may not apply to oscillators which possess a spatial degree of freedom. This result, demonstrated analytically as well as numerically, shows that the additional power output arises from a redistribution of the spatially modulated modes in each oscillator, in response to the interoscillator coupling mechanism. It should therefore be possible to design arrays of long Josephson junctions with considerably enhanced radiation capabilities.

J. B. Hansen is acknowledged for discussions during the initial part of this work. J.A.B. was supported by the Natural Sciences and Engineering Research Council of Canada. This work was performed under the auspices of the U.S. Department of Energy.

[1] D. R. Tilley, Phys. Lett. 33A, 205 (1970).

[2] T. F. Finnegan and S. Wahlsten, Appl. Phys. Lett. 21, $541(1972)$

[3] C. Varmazis, R. D. Sandell, A. K. Jain, and J. E. Lukens, Appl. Phys. Lett. 33, 357 (1978).

[4] A. K. Jain, P. Mankiewich, and J. E. Lukens, Appl. Phys. Lett. 36, 774 (1980).

[5] A. K. Jain, P. M. Mankiewich, A. M. Kadin, R. H. Ono, and J. E. Lukens, IEEE Trans. Mag. 17, 99 (1981).

[6] T. Holst, J. B. Hansen, N. Grønbech-Jensen, and J. A. Blackburn, Phys. Rev. B 42, 127 (1990).

[7] R. Monaco, N. Grønbech-Jensen, and R. D. Parmentier, Phys. Lett. A 151, 195 (1990).

[8] S. Pagano, R. Monaco, and G. Costabile, IEEE Trans. Mag, 25, 1080 (1989).

[9] T. Holst, J. B. Hansen, N. Grønbech-Jensen, and J. A. Blackburn, IEEE Trans. Mag. 27, 2704 (1991).

[10] M. Cirillo, I. Modena, F. Santucci, P. Carelli, and R. Leoni, Phys. Lett. A 167, 175 (1992).

[11] M. B. Mineev, G. S. Mkrtchyan, and V. V. Shmidt, J. Low Temp. Phys. 45, 497 (1981).

[12] Yuri S. Kivshar and Boris A. Malomed, Phys. Rev. B 37, 9325 (1988); Rev. Mod. Phys. 61, 763 (1989).

[13] N. Grønbech-Jensen, M. R. Samuelsen, P. S. Lomdahl, and J. A. Blackburn, Phys. Rev. B 42, 3976 (1990).

[14] D. W. McLaughlin and A. C. Scott, Phys. Rev. A 18, 1652 (1978).

[15] Niels Grønbech-Jensen and Mogens R. Samuelsen (unpublished). 